pachydermatis in an intensive care nursery associated with colonization of health care workers' pet dogs. $N$ Engl J Med 1998;338:706-711.

\section{Clostridium difficile on a Surgical Service}

A study to evaluate the epidemiology of Clostridium difficile colitis in a subset of patients admitted specifically to a surgical service was conducted by investigators from Beth Israel Deaconess Medical Center and Harvard Medical School in Boston, Massachusetts. $C$ difficile colitis is an increasingly prevalent nosocomial infection that can prolong hospitalization and affect patient outcome adversely. Although this disease has been investigated extensively in patients admitted to medical services, the incidence and risk factors for the development of this disease in patients admitted to a surgical service have not been studied.

Over a 5-month period, 21 (5.6\%) of 374 patients admitted to the general, vascular, thoracic, and urologic surgery services developed symptomatic $C$ difficile colitis (defined as more than three bowel movements per 24 hours and a positive cytotoxin assay or culture). Factors that independently predisposed to infection included admission from a skilled-care facility, use of the antibiotic cefoxitin, and an operative procedure for bowel obstruction. Other factors associated with $C$ difficile colitis included colectomy, treatment with any antibiotic, nasogastric tube suction, advanced age, and prior antibiotic treatment. Abdominal pain and fever also were more common in patients with $C$ difficile colitis. Morbidity included prolonged hospitalization in all patients and urgent colectomy in one.

It was concluded that $C$ difficile colitis frequently affects surgical patients, producing morbidity ranging from mild diarrhea to life-threatening illness. A variety of factors, many of which are associated with intestinal stasis, predispose to the development of $C$ difficile colitis.
FROM: Kent KC, Rubin MS, Wroblewski L, Hanff PA, Silen W. The impact of Clostridium difficile on a surgical service: a prospective study of 374 patients. Ann Surg 1998;227:296-301.

\section{Risk of Helicobacter pylori Transmission by Upper Gastrointestinal Endoscopy}

Upper gastrointestinal endoscopy has been reported as a risk factor for the transmission of Helicobacter pylori. Investigators from San Paulo, Brazil, conducted a study to evaluate the possibility of transmission of $H$ pylori infection by upper gastrointestinal endoscopy.

The study included 1,082 patients. Patients who had undergone upper gastrointestinal endoscopy or were treated with antibiotics 15 days before the index endoscopy were excluded. $H$ pylori infection was diagnosed by ultrarapid urease test. Variables analyzed were age, gender, type of dyspepsia (organic or functional), and the number of previous upper gastrointestinal endoscopies.

Patients ranged in age from 13 to 94 years (mean, 45.8; standard deviation [SD], 15.7), and the number of previous upper gastrointestinal endoscopies ranged from 0 to 20 (mean, 1.5; SD, 2.4). The overall prevalence of $H$ pylori infection was $60 \%$. There was no statistically significant difference in the mean number of upper gastrointestinal endoscopies in patients with and without $H$ pylori infection.

The authors concluded that there was no association between history of upper gastrointestinal endoscopy and current $H$ pylori infection in this study population.

FROM: Rohr MR, Castro R, Morais M, Brant CQ, Castelo Filho A, Ferrari AP Jr. Risk of Helicobacter pylori transmission by upper gastrointestinal endoscopy. Am J Infect Control 1998;26:12-15.

Additional news item in this issue: Community-Acquired Methicillin-Resistant Staphylococcus aureus, 322.

NHETIONONIROL HPACIIIONER



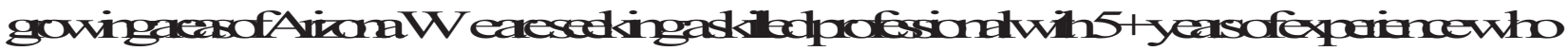

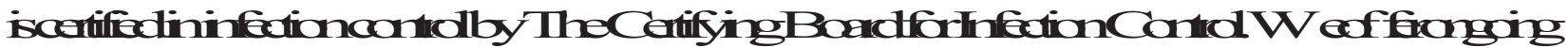



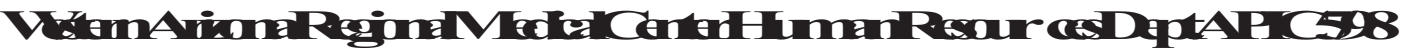
BEslar Trellarad

Bulnead Cy, A78642 FAX520,704634 Wxanduntsubtanceaburetesing KOHMTD. 


\section{REVIEWERS}

Of the many people responsible for the success of Infection Control and Hospital Epidemiology, among the most important are our cadre of reviewers. Their efforts enable us to publish articles of the highest quality, and the editors and authors are grateful for their efforts and advice. You have our deepest thanks.

Jacques Acar
Garrett Adams
J Wesley Alexander
Michelle Alfa
Robert Arbeit
Paul Arnow
Graham Ayliffe
David Bangsberg
Joe Bates
Murray Batt
Nathan Belkin
James Billingsley
Henry Blumberg
Walter Bond
Donald Bornstein
John Boyce
Patrick Brennan
Christian Brun-Buisson
Charles Bryan
John Burke
Jay Butler
Philip Carling
Louisa Chapman
James Cherry
Celia Christie
Stuart Cohen
Donald Craven
Kent Crossley
Lisa Danzig
Franz Daschner
M Delgado-Rodriguez
E Patchen Dellinger
Bradley Doebbeling
Charles Edmiston
Michael Edmond
Theodore Eickhoff
Grace Emori
Javier Ena
Bruce Farber
Barry Farr
Martin Favero
Robert Fekety
D J Flournoy
Patricia Flynn
Sylvia Fonseca
Victoria Fraser
Scott Fridkin
Peter Fuchs
Nelson Gantz
Richard Garibaldi
David L George
Julie Gerberding
Dale Gerding
Aaron Glatt

Donald Goldmann

Barbara Goldrick

Peter Gross

Donna Haiduven

Jo-Ann Harris

David Henderson

Loreen Herwaldt

Peter Heseltine

Karen Hoffman

Rene Holzheimer

Christopher Ingram

Marguerite Jackson

Janine Jagger

John Jernigan

Allen Kaiser

Sheldon Kaplan

Scott Kellerman

Douglas Kernodle

JAJ Kluytmans

Deloris Koziol

Stephen Kritchevsky

Kwan Kew Lai

James Lee

Robert Leggiadro

Ludwig Lettau

Calvin Linnemann

Victor Lorian

Janet Macher

A Macias-Hernandez

Farrin Manian

Walter Marget

Joel Maslow

Glen Mayhall

Allison McGeer

Pat McGovern

John McGowan

Maryanne McGuckin

Jonathan Meakins

Beverly G Mellen

Dick Menzies

Leonard Mermel

Raf Mertens

Kenneth Meyer

Marissa Montecalvo

Robert Muder

Maury Mulligan

Joseph Mylotte

Mary Nettleman

Ellen Neuhaus

Kathy Neuzil

Lindsay Nicolle

Gary Noskin

Juhani Ojajarvi

Jan Evans Patterson
Sindy Paul

Clare Pegues

Lance Peterson

Michael Pfaller

Sandy Pirwitz

Didier Pittet

Vincenzo Puro

Issam Raad

Annette Reboli

Frank Rhame

Bruce Ribner

Evelyn Rodriguez

Manfred Rotter

William Rutala

Lisa Saiman

William Schaffner

William Scheckler

Lynne Sehulster

John Sellick

Kent Sepkowitz

Daniel Sexton

Robert Sherertz

David Shlaes

Robert Shulman

Robert Silva

Bryan Simmons

Andrew Simor

Philip Smith

Denis Spelman

Susan Springthorpe

William Stead

Larry Strausbaugh

Lauri Thrupp

Jerome Tokars

Timothy Townsend

Antoni Trilla

Joan Turner

Ronald Turner

Andreas Voss

Shu-Qun Wang

John Warren

Chatrchi Watanakunakorn

David Weber

John Weems

Robert Weinstein

Wolfgang Weuffen

Sergio Wey

Andreas Widmer

Mary Willey

Edward S Wong

Rebecca Wurtz

Victor Yu

Marcus Zervos 